\title{
A Wavelet Neural Network Based Non-linear Model Predictive Controller for a Multi-variable Coupled Tank System
}

\author{
Kayode Owa ${ }^{1,2} \quad$ Sanjay Sharma ${ }^{1,2} \quad$ Robert Sutton ${ }^{1,2}$ \\ ${ }^{1}$ School of Marine Science and Engineering, University of Plymouth, UK \\ ${ }^{2}$ Marine and Industrial Dynamic Analysis (MIDAS) Research Group, University of Plymouth, UK
}

\begin{abstract}
In this paper, a novel real time non-linear model predictive controller (NMPC) for a multi-variable coupled tank system (CTS) is designed. CTSs are highly non-linear and can be found in many industrial process applications. The involvement of multi-input multi-output (MIMO) system makes the design of an effective controller a challenging task. MIMO systems have inherent couplings, interactions in-between the process input-output variables and generally have an complex internal structure. The aim of this paper is to design, simulate, and implement a novel real time constrained NMPC for a multi-variable CTS with the aid of intelligent system techniques. There are two major formidable challenges hindering the success of the implementation of a NMPC strategy in the MIMO case. The first is the difficulty of obtaining a good non-linear model by training a non-convex complex network to avoid being trapped in a local minimum solution. The second is the online real time optimisation (RTO) of the manipulated variable at every sampling time. A novel wavelet neural network (WNN) with high predicting precision and time-frequency localisation characteristic was selected for an MIMO model and a fast stochastic wavelet gradient algorithm was used for initial training of the network. Furthermore, a genetic algorithm was used to obtain the optimised parameters of the WNN as well as the RTO during the NMPC strategy. The proposed strategy performed well in both simulation and real time on an MIMO CTS. The results indicated that WNN provided better trajectory regulation with less mean-squared-error and average control energy compared to an artificial neural network. It is also shown that the WNN is more robust during abnormal operating conditions.
\end{abstract}

Keywords: Wavelet neural network (WNN), non-linear model predictive control (NMPC), real time practical implementation, multi-input multi-output (MIMO), modelling, system identification, genetic algorithms (GA), non-linear optimisation, coupled tank system (CTS).

\section{Introduction}

Process optimisation is a subject area that deals with the control of processes so as to optimise some stipulated set of parameters and constraints while still maintaining the overall goals and objectives of the plants ${ }^{[1,2]}$. A typical process industry consists of many control loops responsible for controlling parts of the important complex processes such as maintaining level, flow, or temperature. Liquid level control is probably the most common control problem in practical process systems ${ }^{[3]}$. Complex activities in industries can be comprehended easily by the understanding of the basic working principles of some process such as the laboratory coupled tank system $(\mathrm{CTS})^{[4]}$. The diverse utilisations of CTS usages can be found in flow and level controls, temperature controls, chemical blending, reactions vessels, hot-water inputs, storage tanks, and temperature stabilisations $^{[4,5]}$. The usefulness of CTS equipment has also provided many scientists an environment for many re-

\footnotetext{
Regular paper

Manuscript received November 17, 2013; accepted February 14 2014

This work was supported by Petroleum Training Development Fund, Nigeria.

Recommended by Associate Editor Jyh-Horng Chou

(C)Institute of Automation, Chinese Academy of Science and Springer-Verlag Berlin Heidelberg 2015
}

search studies ${ }^{[4,6-13]}$. The coupled tank apparatus is used to analyse and examine the basic and advanced engineering principles which include the study of static and dynamic systems $^{[4,14]}$. A CTS is highly non-linear due to the feature characteristics of the valves and the fundamental dynamic equation which is time variant ${ }^{[3]}$. In the majority of cases, fluids are expected to be pumped and mixed under varying conditions. In all these processes, fluid level, flow and reactants rates must be controlled and regulated ${ }^{[5,15]}$. Real process plants are usually large scale, non-linear, time variant, multi-objective and are very complex ${ }^{[16]}$ and controlling such a multi-input multi-output (MIMO) system poses a very challenging task in the process industries ${ }^{[17]}$. The control of the MIMO processes is more difficult than the single-input single-output (SISO) processes because of the couplings between the process variables. Therefore, the design procedures for SISO systems cannot be used effectively for MIMO systems ${ }^{[18]}$. Many of these processes are complex in nature and exhibit non-linearity and the design of a non-linear MIMO model for the plant is an extremely arduous task ${ }^{[15]}$. Another major design hurdle in the process industries is an improper and an inefficient controller that makes the process runs below its optimum level. A huge economical advantage can be achieved with a design of an optimal control strategy for the process industries ${ }^{[4]}$. 
Classical control strategies such as proportional-integralderivative (PID) have been well established in the process industries $^{[8,10,12,19]}$, but they are not suitable for nonlinear and complex plants. Researchers are always looking for new control methods and approaches for greater and increased efficiency ${ }^{[4]}$. Today, most effective process operations demand operating ranges closer to the boundary of the allowable operating region ${ }^{[1,4]}$ and hence linear models are mostly deficient to sufficiently represent the non-linear dynamics of the plant ${ }^{[4,20]}$. In addition, MIMO control systems are more difficult to design as they integrate multiple sensors data to coordinate multiple actuators. Some researchers used multiple models and combined different controllers for MIMO systems ${ }^{[16,21-22]}$. An accurate non-linear model with an efficient control strategy is thus required to increase efficiency and higher production in the process industries. The model predictive control (MPC) strategy can control and optimise complex processes with constraints ${ }^{[23]}$. Model predictive controllers rely mainly on dynamic models of the process and therefore can either use a linear or nonlinear model representation of the plant for prediction purpose. As already mentioned, process industrial plants are implicitly non-linear in composition ${ }^{[20,24-25]}$ and the prediction performance of MPC becomes complex in the presence of higher degree of non-linearity ${ }^{[25]}$. A reliable and efficient non-linear model is thus required to predict accurately the behaviour of the plant in an MPC strategy. Non-linear model gives a more accurate prediction for a wider operating range of control ${ }^{[4]}$. The couple tank system considered in this study is a typical example of the plant with a high degree of non-linearity ${ }^{[26,27]}$. The non-linearity in the CTS is mainly due to the basic dynamic equations of the CTS, the characteristics of the valves and as a result of the nonlinear flow characteristics in the tank system ${ }^{[4]}$. Most of the literature concerned with CTSs deals with the non-linearity of the SISO system case only ${ }^{[8,28,29]}$ and to the best of the authors knowledge no work has been reported in the literature indicating real-time control of the MIMO system which is recognised as being difficult to achieve. This paper uses a novel approach based on an efficient wavelet neural network (WNN) based model for an MIMO system and the design a non-linear model predictive control (NMPC) strategy for the CTS. This study primarily builds on previous works where a backpropagation artificial neural network $(\mathrm{ANN})$ was used to design NMPC strategies using SISO ${ }^{[4]}$, $\mathrm{MIMO}^{[15]}$ and non-linear CTS models to control the height of the fluid in a second tank. One of the major advantages of using a wavelet for training of a neural network (NN) is the inherent capability of both time and frequency signal localisation, which ultimately helps in achieving a global minimum solution. Wavelets are one of the most exciting research areas in signal processing today and researchers have increasingly seized the opportunity to employ wavelet functions with its choice of different mother wavelet in various modelling disciplines ${ }^{[30-39]}$. The main focus of this work is to use time-frequency localisation feature of the wavelet to design an efficient non-linear MIMO model for a CTS which can operate in nearly all regions of operating points and then design an optimised NMPC for a CTS based on a WNN model. The proposed strategy was tested first in simulation and then in a real time implementation. The results were benchmarked against the NN model in the NMPC and showed that better control actions and accurate control of the CTS were achieved both in terms of mean-squared error (MSE) and in terms of average controller energy (ACE). As no work is recorded yet in the area of robust RTO NMPC on CTS using a single MIMO WNN non-linear model instead of an adaptive model, this paper shows a WNN model to be more robust than an ANN does in the NMPC strategy. The rest of the sections are described as follows: Section 2 describes the CTS while Section 3 contains the modelling details and results of WNN modelling. Section 4 presents the NMPC strategy and a genetic algorithm (GA) real time optimisation (RTO) process. The NMPC strategy results are shown in Section 5 while conclusions are given in Section 6 .

\section{MIMO coupled tank system}

Fig. 1 (a) shows the picture of the multi-variable coupled tank apparatus from TQ TecQuipment CE105MV at Plymouth University while its schematic diagram, which represents a fragment of a typical complex process industry operation, is shown in Fig. 1 (b). A data acquisition (DAQ) device (NI 6009) from National Instrument with LabView software driver is configured to acquire real time sensor data and used to send the multi-variable input to control the fluid levels in both tanks. The algorithms were implemented on a PC with Intel ${ }^{\circledR}$. core $\mathrm{i} 5-2410 \mathrm{M}$ central processing unit (CPU) and 3.0 GB of random-access memory (RAM). The CE105MV unit comprises of two variable speed pumps, two tanks connected by a variable area channel and drain valves to a sump located in the base of the equipment. There are two calibrated piezo-resistive silicon pressure type depth transducers (level sensors), an electronic flow meter and a variable area gap flow meter to provide visual indication of flow rate. The control strategy is designed in a way that the rate of change of the control input is controlled in small steps to avoid major fluctuations. The equipment can be configured as MIMO, SISO, single-input, multi-output (SIMO) or multi-input, single-output (MISO) by the manipulation of pumps inputs and by varying the sectional area of rotary valves $\mathrm{A}$ and $\mathrm{C}$ as shown in Fig. 1 (b). The physical parameters of the TQ CE105MV coupled tank apparatus are given in Table 1. The MIMO configuration adopted in this work involves both pump 1 and pump 2 receiving voltages and pumping fluid into both tanks with valve A fully opened so that there can be interaction between both tanks. In addition, valves $\mathrm{B}$ and $\mathrm{C}$ are opened in midway position with their parameters given in Table 1. The input voltages are also referred to as manipulated variables while the outputs, which are the height or level of the fluid in both tanks, are known as the controlled variables. At any given time, the heights of the fluid in both tanks are related to the fluid inlet rates of both pumps and the outlet rates of both tanks. 


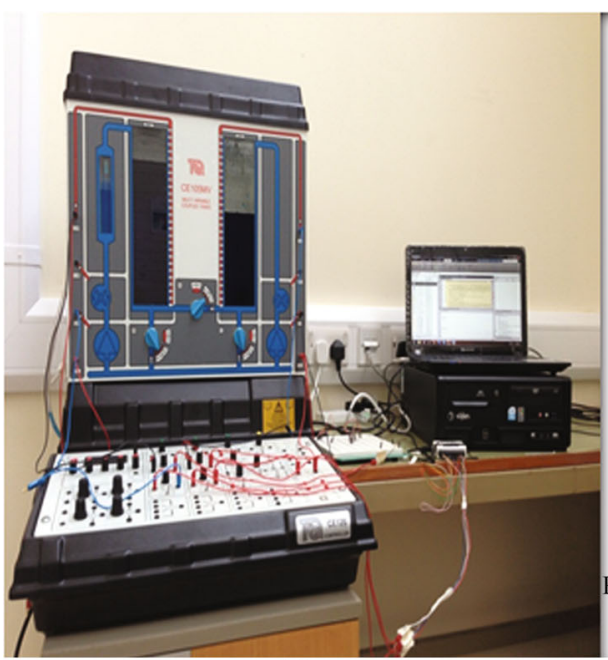

(a)

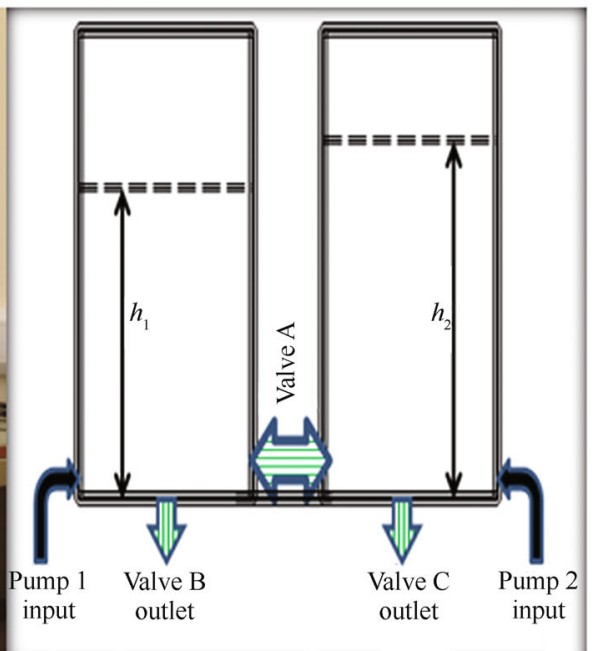

(b)

Fig. 1 Coupled tank system setup. (a) Picture of CTS at Plymouth University; (b) Schematic diagram of CTS

Table 1 Physical parameters of the coupled apparatus

\begin{tabular}{|c|c|c|}
\hline Symbol & Quantity & Value \\
\hline Tank 1 and Tank 2 & Tank cross sectional area & $9.35 \times 10^{-3} \mathrm{~m}^{2}$ \\
\hline Valves $\mathrm{A}\left(\alpha_{12}\right), \mathrm{B}\left(\alpha_{1}\right), \mathrm{C}\left(\alpha_{2}\right)$ & Valve orifice cross sectional area & $7.85 \times 10^{-5} \mathrm{~m}^{2}$ \\
\hline$\beta_{12}$ & Discharge coefficient of $10 \mathrm{~mm}$ valve $A$ orifice between tank 1 and tank 2 & 0.25 \\
\hline$\beta_{1}$ and $\beta_{2}$ & Discharge coefficient of valve $B$ and $C$ orifice & 0.25 \\
\hline$g$ & Gravitational constant & $9.80 \mathrm{~ms}^{-2}$ \\
\hline Liquid level sensors & 0 to $10 \mathrm{~V} \mathrm{DC}$ output (0 to $250 \mathrm{~mm}$ height) & \\
\hline Pump flow sensors & 0 to $10 \mathrm{~V}$ DC output ( 0 to $4400 \mathrm{~cm}^{3} / \mathrm{min}$ ) & \\
\hline
\end{tabular}

\section{System identification and modelling}

The non-linear dynamic equations of the CTS in Fig. 1 are determined by relating the flow $Q_{i}$ into the tank to the flow $Q_{o}$ leaving through the tank valves. Applying the mass balance of flow equation of the tank, it is possible to write ${ }^{[3]}$

$$
Q_{i}-Q_{o}=A \frac{\mathrm{d} h}{\mathrm{~d} t}
$$

where $A$ is the cross-sectional area of the tank, and $h$ is the height of the fluid in the tank. The unit of (1) is expressed in $\mathrm{m}^{3} \cdot \mathrm{s}^{-1}$. The flow through the valve can also be expressed as ${ }^{[3]}$

$$
Q_{o}=\delta_{x} \beta_{x} \alpha_{x} \sqrt{2 g h_{x}}
$$

where $\alpha_{x}$ is the cross sectional area of the orifice, and $\delta_{x}$ is the discharge coefficient of the valve. Note that $\delta_{x}$ takes into account all fluid characteristics, losses and irregularities in the system such that the two sides of the equation balance. In addition, $\beta_{x}$ is the valve opening expressed as ratio. At any given time, the heights of fluid in tank 1 and tank 2 relate to the fluid inlet rates and fluid outlet rates. Therefore, (1) and (2) can be combined together and apply to tank 1 and tank 2 in order to respectively derive

$$
\begin{aligned}
A_{1} \frac{\mathrm{d} h_{1}}{\mathrm{~d} t}= & K_{1} V_{1}(t)-\beta_{1} \alpha_{1} \sqrt{2 g h_{1}} \pm \\
& \beta_{12} \alpha_{12} \sqrt{\left(2 g\left(h_{1}(t)-h_{2}(t)\right)\right)}
\end{aligned}
$$

$$
\begin{aligned}
A_{2} \frac{\mathrm{d} h_{2}}{\mathrm{~d} t}= & K_{2} V_{2}(t)-\beta_{2} \alpha_{2} \sqrt{2 g h_{2}} \pm \\
& \beta_{12} \alpha_{12} \sqrt{\left(2 g\left(h_{1}(t)-h_{2}(t)\right)\right)}
\end{aligned}
$$

where $A_{1}$ and $A_{2}$ are the cross sectional areas of tanks, $h_{1}$ and $h_{2}$ are the fluid levels of the tanks. $K_{1}$ and $K_{2}$ are respective constants of the pumps and the units are expressed in $\mathrm{m}^{3} \mathrm{~s}^{-1} \mathrm{~V}^{-1}$. Subscripts 1 and 2 refer to tanks 1 and 2, respectively. The discharge coefficient of the valve takes into account the fluid characteristics, losses and irregularities in the system such that the two sides of the equation balance and cancel out. The rest of the parameters are given in Table 1. Fig. 2 shows the Simulink representation of the MIMO coupled tank equations, where input 1 is $u_{1}$, input 2 is $u_{2}$, output 1 is $h_{1}$ and output 2 is $h_{2}$. This representation of the plant will be used in simulation to test the NMPC algorithm.

System identification techniques are used to predict the behaviour of the CTS using the raw measured input output data. Three sets of different input-output data of 2445 sample each were collected from real open loop practical experiments on the MIMO coupled tank system with a sampling rate $T s$ of $0.2 \mathrm{~s}$. Figs. $3(\mathrm{a})-(\mathrm{c})$ show the three data sets collected for analysis. These are crucial plant details for system identification stage. These samples of data were collected and obtained in such a way as to show the clear differences in the two output variables, the fluid filling up and draining processes of the two output variables. 


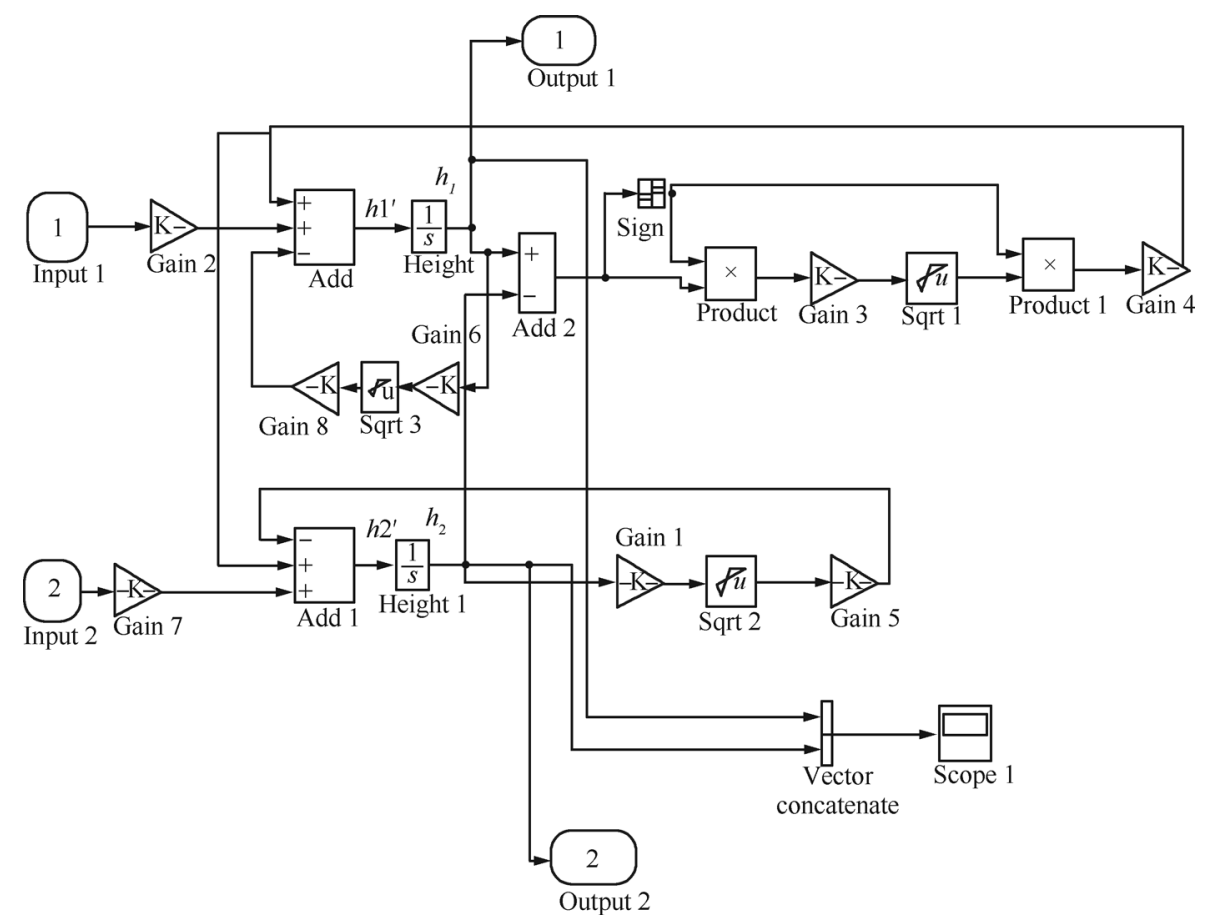

Fig. 2 MIMO coupled tank system in Simulink ${ }^{\circledR}$ design

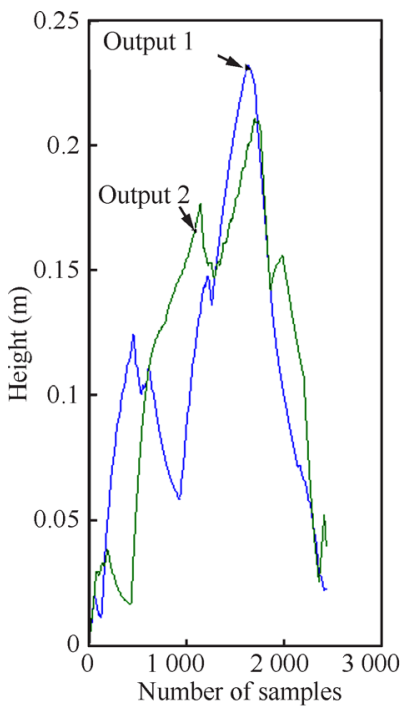

(a) Training data

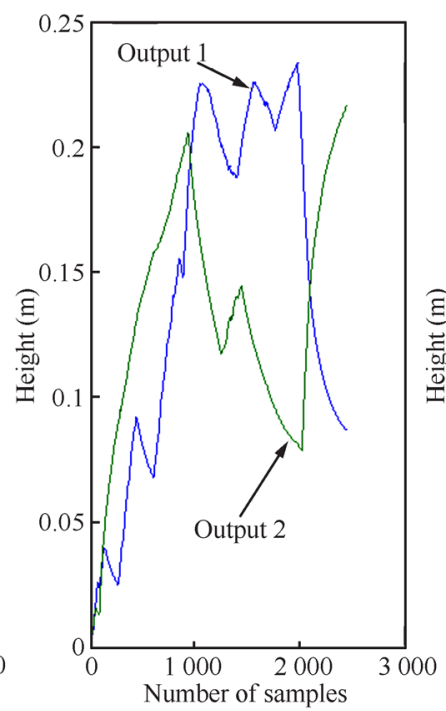

(b) Validation data

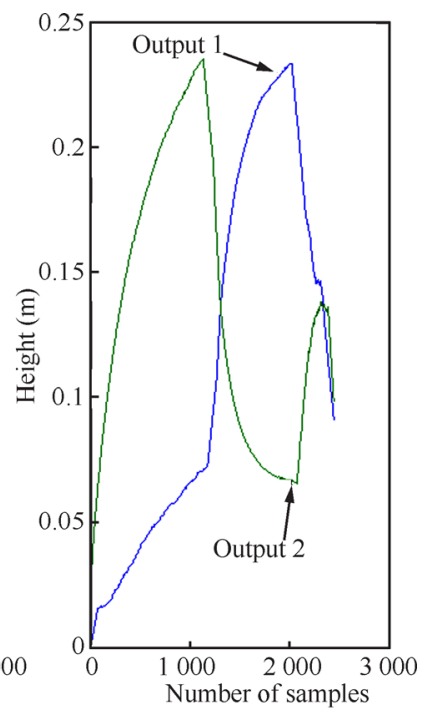

(c) Test data

Fig. 3 Data collected for analysis

\subsection{Wavelet neural network (WNN) for modelling of the CTS}

The WNN has wavelets functions in the hidden layer which is also referred to as a wavelet layer. Training of a WNN involves finding the unknown weights between input to hidden layer $\left(W I_{j i}\right)$, hidden to output layer $\left(W O_{k j}\right)$, translation factor $\left(b_{j}\right)$ and dilation (expansion) factor $\left(a_{j}\right)$. For further details of WNN, the intended reader is referred to [40]. In this work, a Morlet wavelet $\varphi_{x}$ is selected as a mother wavelet. The wavelet $\varphi_{x}$ is expressed in (5) and is used as the activation function for the neurons in the hidden layers of the WNN.

$$
\varphi(x)=\cos (1.75 x) \times \mathrm{e}^{-\frac{x^{2}}{2}}
$$

where 1.75 is the modulation factor and $x$ is expressed as

$$
x=\frac{\sum_{i=1}^{L}\left(W I_{j i} X_{i}^{n}-b_{j}\right)}{a_{j}} .
$$

A wavelet transform allows exceptional localisation in the time domain via translation (a shifting process) and also in 
the frequency domain via dilation (a scaling process) of the mother wavelet. The effect of this shifting and scaling process is to produce a time-frequency representation of the signal. The wavelet basis functions are shifted in time domain to maintain the same number of oscillations and its frequency scaled in amplitude to maintain energy. Owing to their capability to localise in time, wavelet transforms readily lend themselves to non-stationary signal analysis. The architecture of an MIMO WNN is shown in Fig. 4, where the inclusion of wavelet activation functions in the hidden layer increases the number of unknown in WNN training compared to a traditional ANN. An initial heuristics study was conducted to ascertain the optimal number of parameters of the WNN, which is represented in a non-linear auto-regression with exogeneous, inputs (NARX) form of $y_{\text {model }}=f\left(h_{2}(t-1), h_{2}(t-2), h_{1}(t-1), h_{1}(t-2), u_{1}(t), u_{1}(t-\right.$ $\left.1), u_{2}(t), u_{2}(t-1)\right)$, where $f(\cdot)$ is an unknown complex nonlinear function. Here, 2 hidden neurons with two input and two output delays, which give a total of 24 unknown parameters in the WNN structure, were chosen after many initial trials of different values and configurations. The training data set consists of two sequences of vectors, which is the total number of samples of input sequences $u_{1}$ and $u_{2}$ and measured process outputs $h_{1}$ and $h_{2}$. These are arranged in a regressed form of the specified number of two-input two-output delay feedback. The aim here is to create a WNN model by finding the optimised unknown parameters as expressed in (7), where the WNN model output is $y_{\text {model }}$.

$$
y_{\text {model }}(t)=\sum_{j=1}^{N} \prod_{i=1}^{P} w_{i} \varphi_{i}+\sum_{i=1}^{S} \prod_{o=1}^{Q} \text { net }_{i} w_{o}
$$

where $n e t_{i}$ is the net value of each of the hidden neurons $i$.

The term $\frac{\delta \varphi_{x}}{\delta_{x}}$ in (8) is the derivative of (5). This will be used as part of the terms for calculating the partial deriva- tives of the error $\epsilon$ functions in (9).

$$
\frac{\partial \varphi(x)}{\partial x}=-\{x \cos (1.75 x)+1.75 \sin (1.75 x)\} \times \mathrm{e}^{\frac{x^{2}}{2}} .
$$

The partial derivatives of the unknown weights $W I_{j i}$, $W O_{k j}, b_{j}$ and $a_{j}$ are calculated in (9) by using a conjugate stochastic gradient method:

$$
\begin{gathered}
\frac{\partial \xi}{\partial W I_{j i}}=\frac{1}{N} \sum_{n=1}^{N} \sum_{k=1}^{S}\left\{\left(D_{k}^{n}-Y_{k}^{n}\right) \times W O_{k J} \times \frac{\partial \varphi(x)}{\partial x} \frac{X_{i}^{n}}{a_{j}}\right\} \\
\frac{\partial \xi}{\partial W O_{k j}}=\frac{1}{N} \sum_{n=1}^{N}\left(D_{k}^{n}-Y_{k}^{n}\right) \times \varphi\left\{\frac{\sum_{i=1}^{L}\left(W I_{j i} X_{i}^{n}-b_{j}\right)}{a_{j}}\right\} \\
\frac{\partial \xi}{\partial b_{j}}=\frac{1}{N} \sum_{n=1}^{N} \sum_{k=1}^{S}\left(D_{k}^{n}-Y_{k}^{n}\right) \times W O_{k J} \times \frac{\partial \varphi(x)}{\partial x} \times \frac{1}{a_{j}} \\
\frac{\partial \xi}{\partial a_{j}}=\frac{1}{N} \sum_{n=1}^{N} \sum_{k=1}^{S}\left(D_{k}^{n}-Y_{k}^{n}\right) \times W O_{k J} \times \\
\frac{\partial \varphi(x)}{\partial x} \times\left\{\frac{\sum_{i=1}^{L}\left(W I_{j i} X_{i}^{n}-b_{j}\right)}{a_{j}^{2}}\right\}
\end{gathered}
$$

where $\xi$ is the partial derivative of the error, $N$ is the number of samples to be trained, $S$ is the number of outputs, $L$ is the number of regressed inputs in the WNN structure. In addition, $D$ in (9) refers to $y_{\text {model }}$ while $Y$ is used to represent $y_{\text {target }}$. The partial derivatives are subsequently used to update the unknown weights using the formulae:

$$
\begin{aligned}
& W I_{j i}^{i i+1}=W I_{j i}^{i i}-\eta_{j j} \times \frac{\partial \xi}{\partial W I_{j i}^{i i}} \\
& W O_{k j}^{i i+1}=W O_{k j}^{i i}-\eta_{j j} \times \frac{\partial \xi}{\partial W O_{k j}^{i i}} \\
& b_{j}^{i i+1}=b_{j}^{i i}-\eta_{j j} \times \frac{\partial \xi}{\partial b_{j}^{i i}} \\
& a_{j}^{i i+1}=a_{j}^{i i}-\eta_{j j} \times \frac{\partial \xi}{\partial a_{j}^{i i}} .
\end{aligned}
$$

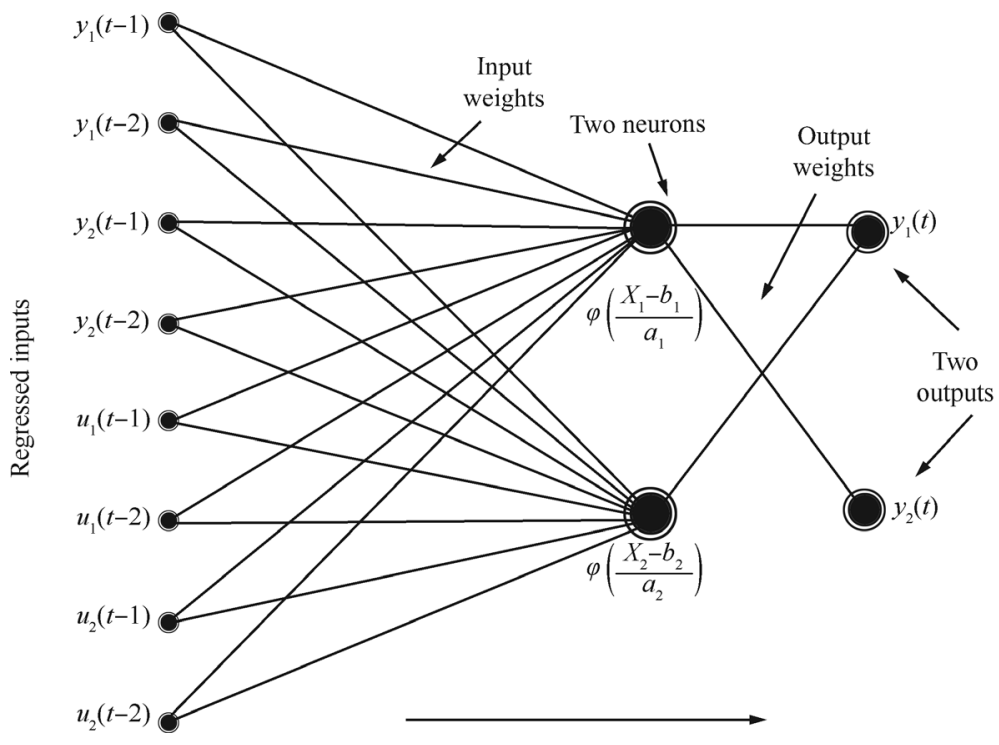

Fig. 4 Structure of a conventional MIMO WNN 
The training of the feed forward WNN is based on the minimisation of the error between the model and the target as shown below:

$$
\mathrm{MSE}=\sum_{n=1}^{N} \sum_{k=1}^{S} \frac{\left(y_{\text {model } k}^{n}-y_{\text {target }}^{n}\right)^{2}}{N}=\sum_{n=1}^{N} \frac{\mathrm{e}^{n^{2}}}{N} .
$$

During the training process, the validation process is constantly carried out. The WNN is initially trained using the stochastic conjugate gradient method and the training stops immediately when the validation model error value starts to increase. The optimal weight derived from this stage is used to generate an initial 100 population for a GA, which was run for 500 generations to obtain the optimal values of the unknown parameters of the WNN. This is a case of non-linear MIMO system, which is more challenging than most recently reported SISO in literature ${ }^{[8,18,28,41]}$, and the training approach was adopted for the following reasons in order to avoid the problem of being trapped in a local minimum solution. Moreover, it was shown after some heuristic study that the proposed is a faster approach. The ANN model is also obtained in a similar manner for benchmarking purpose.

\subsection{Modelling results}

The results of the modelling are given in Table 2. The mean square errors (MSE) for both ANN and WNN are calculated using (11). The MSEs were calculated for the training, validation and the test data. WNN gave lower MSEs as compared to ANN in all the three data collected. The means and the variances of the two-input signals for the three data samples used to excite the real plant are also given in Table 2. Figs. 5 and 6 give the characteristics and performance modelling results of ANN and WNN, respectively. Figs. $5(\mathrm{a})$ and 6 (a) are the plots of both real plant output and the model output for the first output and their prediction errors while Figs. 5 (b) and 6 (b) are similarly the plots of both real plant output and the model output for the second output and their prediction errors. These are the one step ahead prediction generated by both derived models. Their MSEs values are already given in Table 2. Furthermore, Figs. 5 (c), 5 (d), 6 (c), and 6 (d) are the plots of the auto-correlation and the cross-correlation model results. Figs. 5 (c) and 6 (c) represent the first output while Figs. $5(\mathrm{~d})$ and $6(\mathrm{~d})$ show the second output results for the ANN and WNN models, respectively. For the autocorrelation results, the ANN model results in Figs. 5 (c) and 5 (d) are the auto-correlation coefficients graphs of the prediction errors for both outputs 1 and 2, respectively. They do not have any value close to zero or within $10 \%$ confidence intervals. However, the auto-correlation coefficient of the WNN prediction errors in Fig. 6 (c) (outputs 1) and Fig. 6 (d) (output 2) have $50 \%$ more zero values in the $10 \%$ confidence intervals than the ANN model. This is a measure of the validation of the network performance and it gives the indication of how the prediction errors are related in time. For a perfect prediction model using the auto-correlation function, there should only be one non-zero value which should occur at the zero lag. This would mean that the prediction errors were completely uncorrelated with each other.

For the cross-correlation results, both the ANN and WNN cases have their cross-correlation coefficients inputs fall with the confidence intervals. Also, for a perfect prediction model using the cross-correlation function, all of the correlations should be zero. Some initials heuristics trials were carried out which indicated that the model results will be admissible if the error correlations fall within the $10 \%$ confidence interval boundaries around zero. Therefore in this paper, the derived models that fall within the specified confidence intervals range performed excellently in the NMPC strategy. The aim of the correlation results is to make sure that the model is so good for prediction purpose that there would not be correlation within the prediction errors.

\section{Non-linear control strategy for the CTS}

NMPC is an advanced control strategy in which the currently manipulated control input applies to the real plant. A finite prediction horizon ( $\mathrm{PH})$ open-loop optimal control problem is derived by obtaining a real time solution online at each sampling instant using a non-linear MIMO model for prediction. The optimisation yields an optimal control sequence and the first control in this sequence is applied to the plant. A schematic picture of the whole control strategy is shown in Fig. 7. The predictor task is to predict the plant output based on the regressed inputs at every instant. This is done for different control moves within a prediction range. The value of the control horizon $(\mathrm{CH})$ should always be less than the PH. The model predictive control strategy was implemented by using a GA in real time to solve and minimise the complex optimisation cost function (12) at every sampling time. This is used to determine the best or optimum control inputs that give the least error between

Table 2 Modelling results for both ANN and WNN

\begin{tabular}{|c|c|c|c|c|c|c|}
\hline \multirow{2}{*}{$\frac{\text { Performance function (outputs) }}{\text { ANN-MSE }\left(\mathrm{m}^{2}\right)^{[15]}}$} & \multicolumn{2}{|c|}{ Data one (training) } & \multicolumn{2}{|c|}{ Data two (validation) } & \multicolumn{2}{|c|}{ Data three (testing) } \\
\hline & & $3395 \times 10^{-6}$ & & $7.0135 \times 10^{-6}$ & & $2.8588 \times 10^{-5}$ \\
\hline WNN-MSE $\left(\mathrm{m}^{2}\right)$ [Proposed] & & $1331 \times 10^{-8}$ & & $9.1679 \times 10^{-8}$ & & $1.7239 \times 10^{-7}$ \\
\hline Inputs & Input 1 & Input 2 & Input 1 & Input 2 & Input 1 & Input 2 \\
\hline Mean (V) & 4.0851 & 5.0091 & 4.8336 & 4.9532 & 4.5705 & 5.3594 \\
\hline Variance (V) & 9.1842 & 6.9189 & 11.5329 & 14.3033 & 11.0491 & 14.5067 \\
\hline
\end{tabular}


the predicted output and the trajectory reference signals and minimise the controller efforts while the predictor is the non-linear WNN model.

$$
\begin{aligned}
J(\theta)= & \sum_{i=1}^{P} \sum_{j=1}^{P}\left|w_{i+1}^{y}\left(y_{j}(k+i+1 \mid k)-r_{j}(k+i+1)\right)\right|^{2}+ \\
& \left.\sum_{j=1}^{n_{u}}\left|w_{i, j}^{\Delta} u \Delta u_{j}(k+i \mid k)\right|^{2}\right) .
\end{aligned}
$$

The value $J(\theta)$ on the left-hand side (LHS) of (12) is a one dimensional vector result of the solution of optimisation problem using a GA. The length of vector $J$ is equivalent to the population size (PS) in the GA algorithm. The task is to select the particular population chromosomes from the optimised vectors $(\mathrm{PS} \times($ number of outputs $\times \mathrm{CH}))$ that corresponds to the entry that has the least error value in vector $J(\theta)$ at every sampling instant. The first value in the control horizon of each output is then applied to the
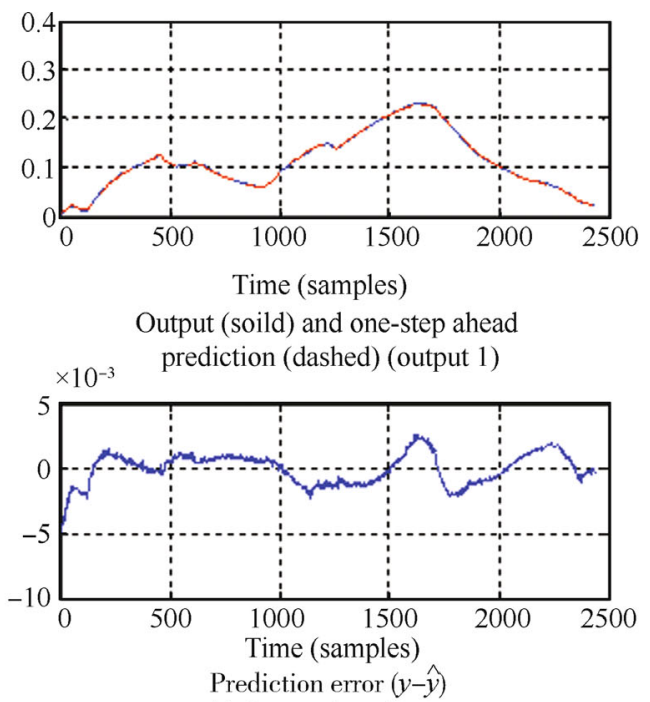

(a) Output 1 prediction

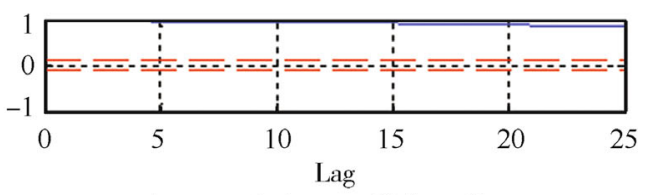

Autocorrelation coefficients for prediction error (output 1)

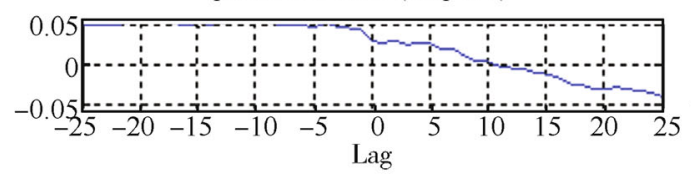

Cross-correlation coef. of $u_{1}$ and prediction error

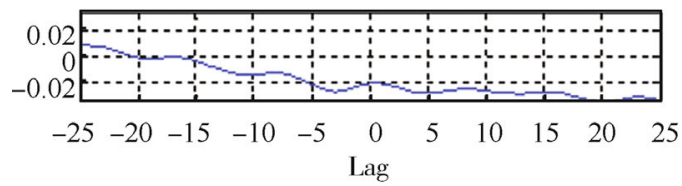

Cross-correlstion coef. of $u_{2}$ and prediction error

(c) Autocorrelation and cross-correlation of output 1 plant. In addition, the first summation on the right-hand side (RHS) of (12) represents the error in prediction value and the reference valve while the second summation term denotes the change in the controller actions which are the previous and current manipulated variables $\left(u_{j+1}-u_{j}\right)$. These are then calculated from the GA optimised manipulated variables. These parameters are the bounded randomly generated population in the GA. The values $r_{j}$ and $y_{j}$ stand for the reference value and plant output, respectively while, $w^{i}$ stands for the weight value. The parameters in (12) that represent the randomly generated population in the GA are the manipulated variables in vector form, which represents the controller actions from pump 1, and pump 2 , respectively. In order to deal with real-time implementation constraints, termination measures were implemented to abort the optimisation once a defined sampling time is passed. This invariably leads to convergence to some suboptimal/optimal solution within the sampling time period.

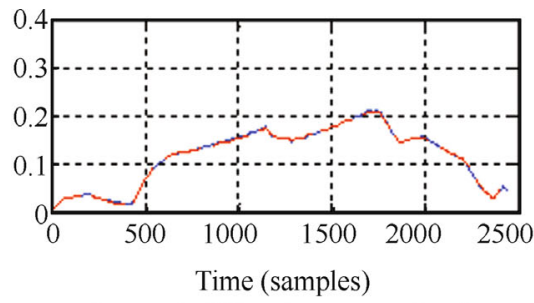

Output (soild) and one-step ahasd prediction (dashed) (output 2)

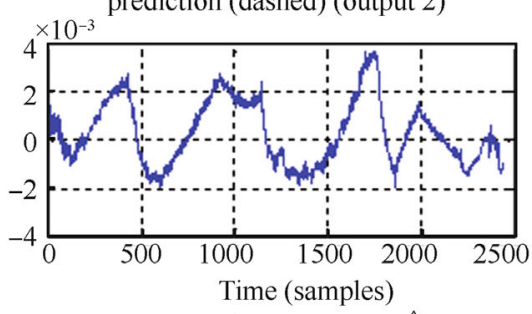

Prediction error $(y-\hat{y})$

(b) Output 2 prediction

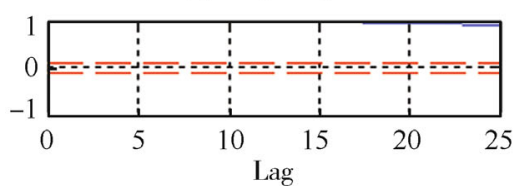

Autocorrelation coefficients for prediction error (output 2)

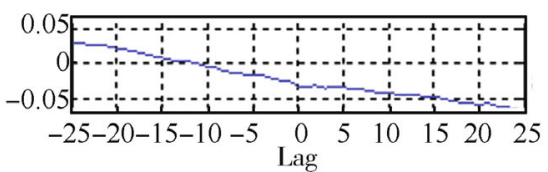

Cross-correlation coef. of $u_{1}$ and prediction error

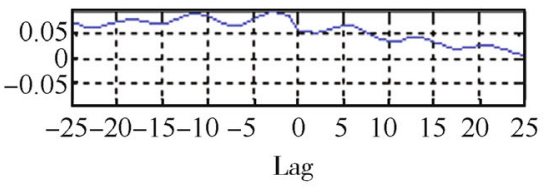

Cross-correlstion coef. of $\boldsymbol{u}_{2}$ and prediction error (d) Autocorrelation and cross-correlation of output 2

Fig. 5 ANN model results 


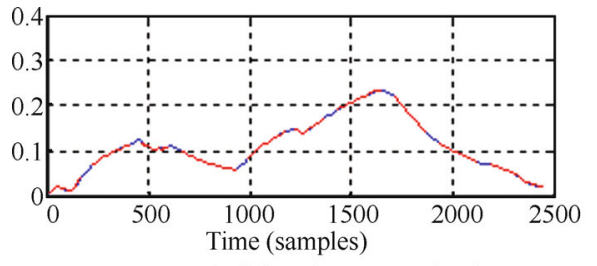

Output (soild) and one-step ahasd

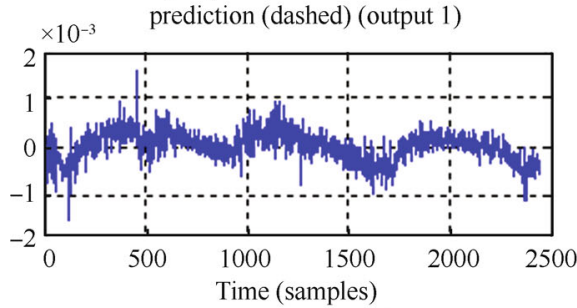

Prediction error $(y-\hat{y})$

(a) Output 1 prediction

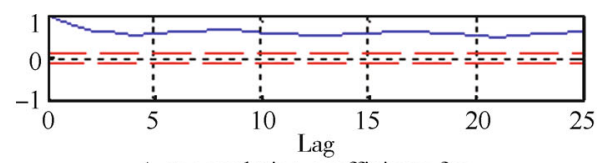

Autocorrelation coefficients for prediction error (output 1)

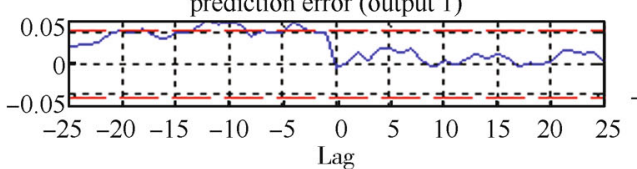

Cross-correlation coef. of $u_{1}$ and prediction error

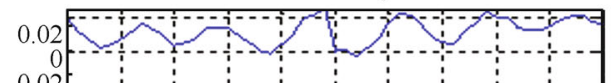

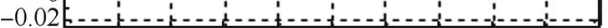

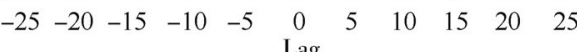

Cross-correlation coef. of $u_{2}$ and prediction error

Cross-correlstion coef. of $u_{2}$ and prediction error

(c) Autocorrelation and cross-correlation of output 1

(d) Autocorrelation and cross-correlation of output 2

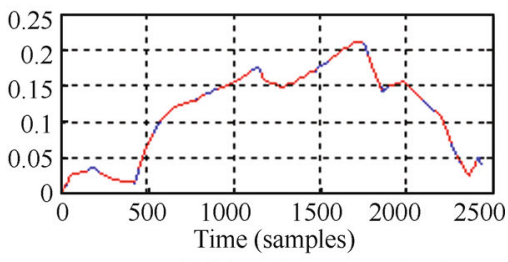

Output (soild) and one-step ahasd $\times 10^{-3}$ prediction (dashed) (output 2)

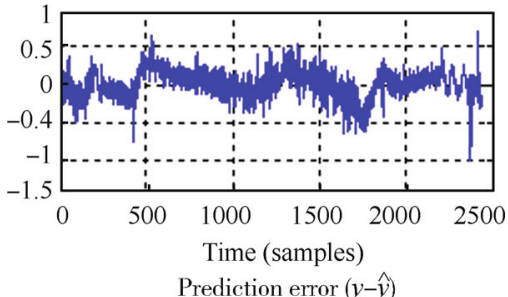

(b) Output 2 prediction

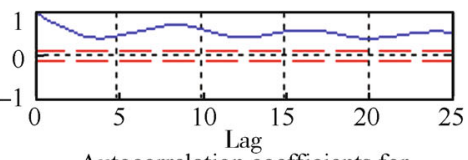

Autocorrelation coefficients for prediction error (output 2)

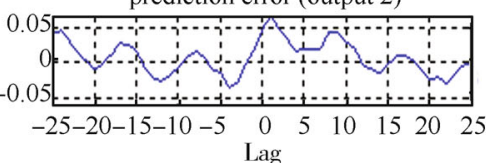

Cross-correlation coef. of $u_{1}$ and prediction error

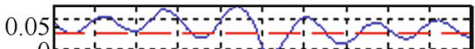

00

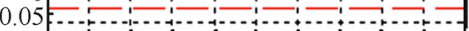

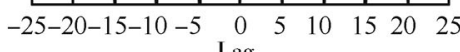
La

Fig. 6 WNN model results

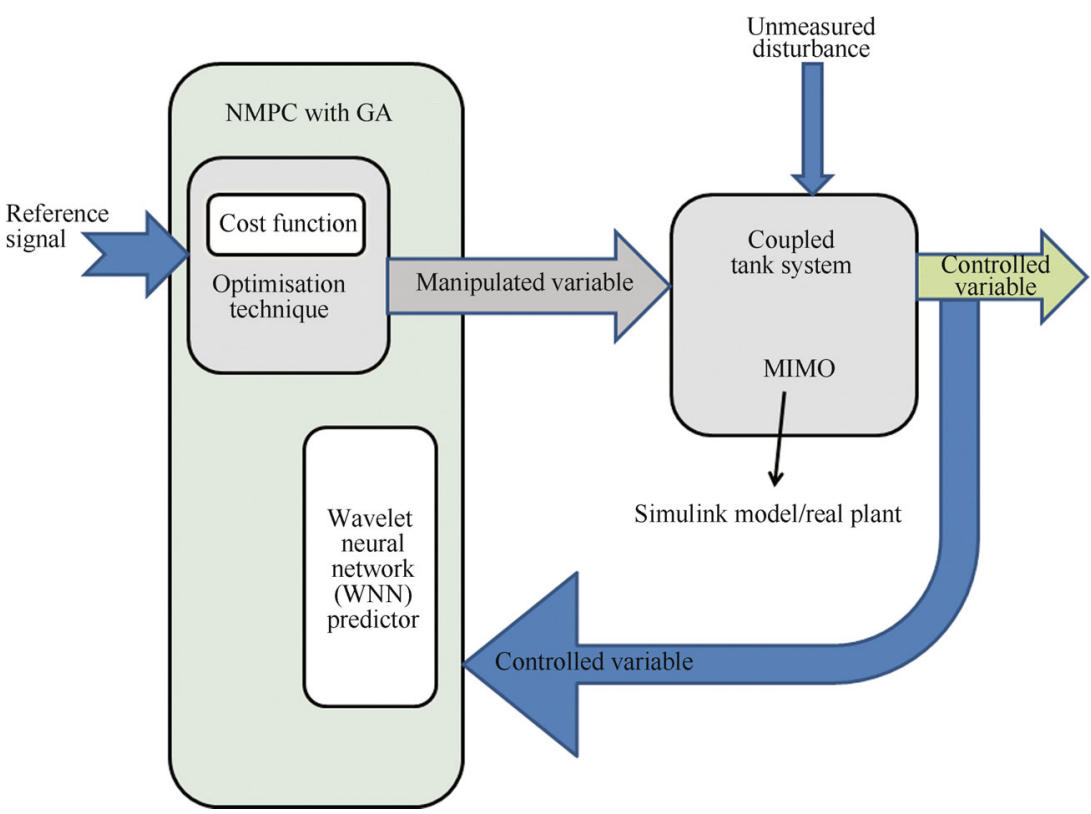

Fig. 7 Structure of the NMPC strategy with GA optimisation 


\subsection{Genetic algorithm}

The GA is a stochastic global search method that operates on a population of potential solutions applying the principle of survival of the fittest to evolve a better candidate to a solution. Here a GA is used to obtain a sequence of optimal manipulated variable control signals that operate the plant. The flowchart for the process involved in the genetic algorithm is shown in Fig. 8. In this work, realvalued genes are used to represent population chromosomes as they provide faster optimisation as real-valued genes use less memory and there is no need to convert chromosomes to phenotypes before each function evaluation. Initial populations are generated randomly between the range of 0 and $10 \mathrm{~V}$. This population is created so that the difference between consecutive $\mathrm{CHs}$ is not more than a prescribed value of $1.5 \mathrm{~V}$. These are constraints limiting the range of control signal whereas the difference between the individual control inputs into the plant limits the gradient of the control signal. In the case of a minimisation problem, the best individuals will have the lowest numerical value of the associated objective function. Individuals are assigned fitness values according to their ranks in the population in each generation before selections are made. The fitness value is calculated using

$$
\text { fitness }=\frac{1}{J+1} .
$$

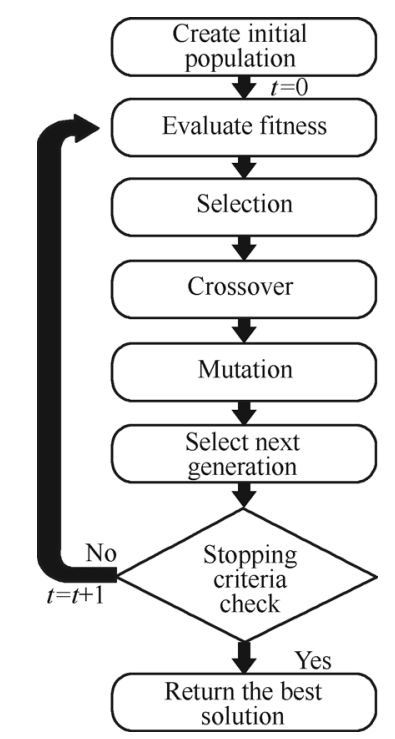

Fig. 8 Flowchart of a genetic algorithm procedure
Mutation brings variations, diversities and changes in the genetic structures of the overall population while crossover process interchanges the genetic structure of two or more chromosomes.

The modified NMPC algorithm is written in such a way that during the constrained optimisation process, the best pairs of $\mathrm{CH}$ vector (population) is constantly retained so that the best population is not destroyed. The best population is constantly preserved from one generation to the other. After some heuristic trials, 0.5 and 0.05 were respectively chosen for crossover and mutation ratios. The GA optimisation parameters ( $\mathrm{PS}=10, \mathrm{PH}=5, \mathrm{CH}=2$ and a generation $=20)$ and model (neurons $=2$, and delays $=2$ ) parameters are carefully chosen in order to guarantee suboptimal/optimal solution at every sampling instant during the NMPC strategy.

\section{Results and discussion}

The result of the design of an ANN model in previous work $^{[15]}$ and the proposed WNN model are given in Table 2. The MSE is used as the performance criteria. Two performance indexes are considered here to evaluate the performance of the NMPC strategy: the MSE in (14) and the average control energy (ACE) in (15). The MSE is the addition of all the squares of the error differences between the reference and the plant output for the two outputs divided by the total number of samples.

$$
\mathrm{MSE}=\frac{\sum_{j=1}^{N}\left(y_{1}^{r}{ }_{j}^{r}-y_{1}^{p}{ }_{j}^{p}\right)^{2}+\sum_{j=1}^{N}\left(y_{2}^{r}{ }_{j}^{r}-y_{2}^{p}{ }_{j}^{2}\right.}{N} .
$$

In (14), superscripts $r$ and $p$ stand for the reference value and plant output, respectively, while $N$ stands for the total number of samples. The average control energy is defined as the addition of the squares of all the manipulated variable inputs $\left(U_{1}\right.$ and $\left.U_{2}\right)$ to the plant divided by the total number of samples and expressed as

$$
\mathrm{ACE}=\frac{\sum_{j=1}^{N} U_{1 j}^{2}+\sum_{j=1}^{N} U_{2 j}^{2}}{N} .
$$

The performance of the NMPC strategy are analysed in simulation with Simulink model as plant. All the NMPC

\begin{tabular}{|c|c|c|c|c|c|}
\hline \multirow[b]{2}{*}{ Scenarios } & & \multicolumn{2}{|c|}{ Simulation } & \multicolumn{2}{|c|}{ Real time } \\
\hline & & ANN & WNN & $\mathrm{ANN}$ & WNN \\
\hline \multirow{2}{*}{ All heights (Figs. 9 and 10) } & $\operatorname{MSE}\left(\mathrm{m}^{2}\right)$ & 0.0049 & 0.0046 & 0.0036 & 0.0022 \\
\hline & $\operatorname{ACE}\left(\mathrm{V}^{2}\right)$ & 82.41 & 78.26 & 55.01 & 65.92 \\
\hline \multirow{2}{*}{ Abnormal condition at lower height (Figs. 11 and 12) } & $\operatorname{MSE}\left(\mathrm{m}^{2}\right)$ & 0.0123 & 0.0122 & 0.004 & 0.0037 \\
\hline & $\operatorname{ACE}\left(V^{2}\right)$ & 10.45 & 9.96 & 135.41 & 127.88 \\
\hline \multirow{2}{*}{ Abnormal condition at upper height (Figs. 13 and 14) } & $\operatorname{MSE}\left(\mathrm{m}^{2}\right)$ & $3.1 \times 10^{-4}$ & $8.2 \times 10^{-5}$ & 0.0048 & $1.4 \times 10^{-4}$ \\
\hline & $\mathrm{ACE}\left(\mathrm{V}^{2}\right)$ & 67.53 & 90.72 & 76.13 & 84.46 \\
\hline
\end{tabular}
strategy results are given in Table 3 .

Table 3 NMPC strategy results for both ANN and WNN 
Fig. 9 gives the response and the comparisons of the NMPC strategies for the simulation of both ANN and WNN, respectively. These show the ability to track all different heights up to the maximum of $0.25 \mathrm{~m}$. The MSE obtained for Fig. 9 (a) is $0.0049 \mathrm{~m}^{2}$ (ANN) and Fig. 9 (b) is $0.0046 \mathrm{~m}^{2}(\mathrm{WNN})$ while their ACE values are $82.41 \mathrm{~V}^{2}$ and $78.26 \mathrm{~V}^{2}$, respectively.

The WNN-NMPC strategy has a better energy usage and less MSE as compared to that of ANN-NMPC. In addition, the WNN-NMPC strategy is able to track precisely the maximum height without tank spillage unlike the ANNNMPC strategy. These results show that the WNN-NMPC requires lower average control energy in order to pump and control different heights. The same experiment is performed in real time and the response result is shown in Fig. 10. The real time result for the WNN-NMPC strategy is also better as in the simulation case. The WNN has lower MSE of $0.0046 \mathrm{~m}^{2}$ as compared to $0.0049 \mathrm{~m}^{2}$ for ANN. It can be seen from Table 3 that $\mathrm{ANN}$ has a lower $\mathrm{ACE}$ value of $55.01 \mathrm{~V}^{2}$ than $65.92 \mathrm{~V}^{2}$ for WNN. The rational reason for this ACE result is that ANN-NMPC strategy required lower work to be expended in order to carry out the inefficient and imprecise trajectory tracking unlike in the case of the WNN-NMPC strategy.

The plant is further subjected to two different abnormal scenarios by increasing the interaction between the two tanks and the opening of both outlet valves for the two tanks. This creates a different scenario as compared with the data obtained for system identification. In the first scenario, fluid is first pumped to the levels $0.25 \mathrm{~m}$ and $0.20 \mathrm{~m}$ for tanks 1 and 2, respectively. From the start of the experiment, three valves $\mathrm{A}, \mathrm{B}$ and $\mathrm{C}$ are all left fully opened to the maximum and the NMPC strategy was used to control the lower heights of the fluid in both tanks. Figs. 11-12 ((a) and (b)) show the performances of the NMPC strategies for both simulation and real time implementation. It can be observed that the NMPC strategy was able to control the low heights with both models. In this case, WNN also has lower values for both $\operatorname{MSE}\left(0.0122 \mathrm{~m}^{2}\right)$ and $\operatorname{ACE}\left(9.96 \mathrm{~V}^{2}\right)$ than $\mathrm{ANN}$ with $\operatorname{MSE}\left(0.0123 \mathrm{~m}^{2}\right)$ and ACE $\left(10.45 \mathrm{~V}^{2}\right)$.

In the second scenario, the tanks contain initial pumped fluid as done previously. From the start of the experiment, three valves $\mathrm{A}, \mathrm{B}$ and $\mathrm{C}$ are all left fully closed and the NMPC strategy was used to control the same high height of the fluid in each tank. Precisely, after $40 \mathrm{~s}$ of operation, valves $\mathrm{B}$ and $\mathrm{C}$ are fully opened to create abnormal situation of valves failure. Four seconds later valve $\mathrm{A}$ is also fully opened to give a maximum interaction, which also creates different dynamics from the previously trained data. Figs. 13 (a) and (b) show the simulation performances of the the NMPC strategy with both ANN and WNN models in the abnormal situation of tracking high height. WNN has MSE value of $8.2 \times 10^{-5} \mathrm{~m}^{2}$ and ACE of $90.72 \mathrm{~V}^{2}$ with a better performance than ANN. WNN-NMPC strategy was able to maintain the same initial height with WNN but ANN model has a steady state. In this case, it can be observed from Table 3 that ANN however has a lower ACE of $67.53 \mathrm{~V}^{2}$ than WNN. This is because ANN-NMPC completely failed to control the level of fluid at the desired heights. It shows further that the WNN-NMPC strategy is reliable and robust. This is useful in abnormal situations like valve malfunctions or total valve failure.

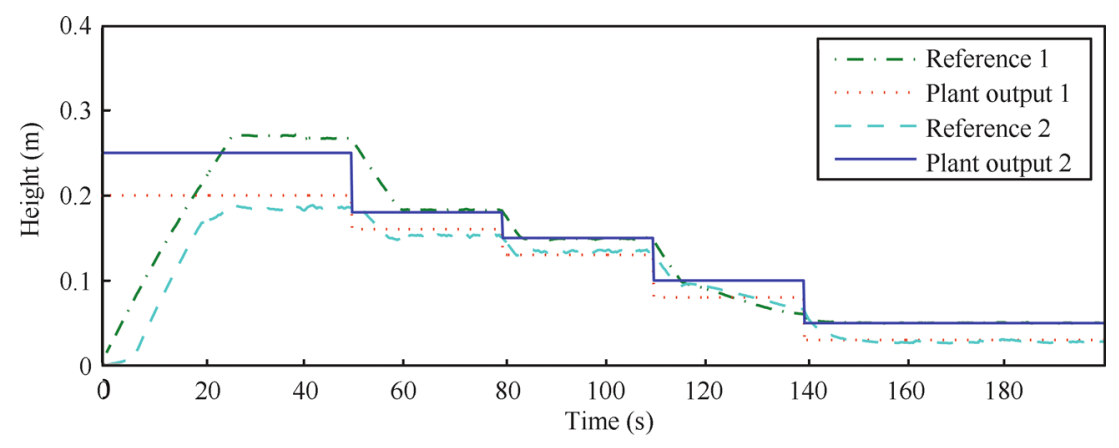

(a) ANN

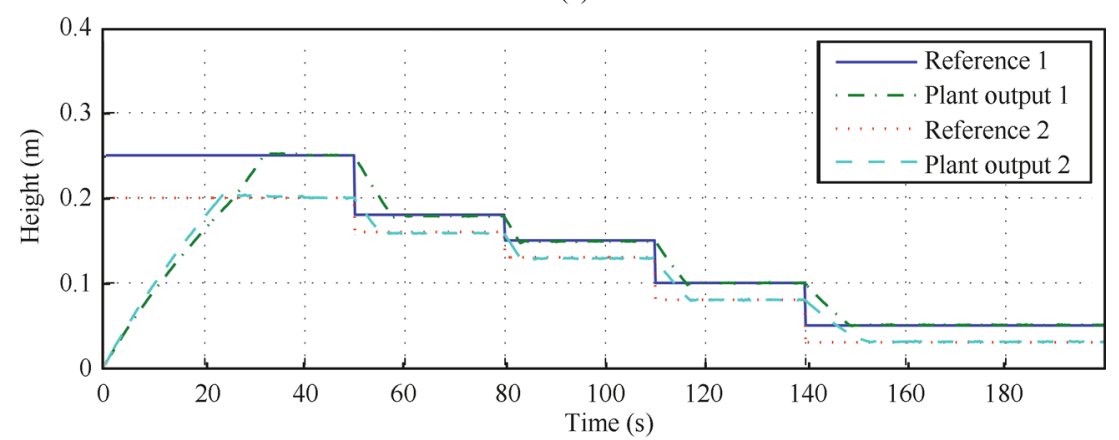

(b) WNN

Fig. 9 Simulation of NMPC strategy tracking different heights 


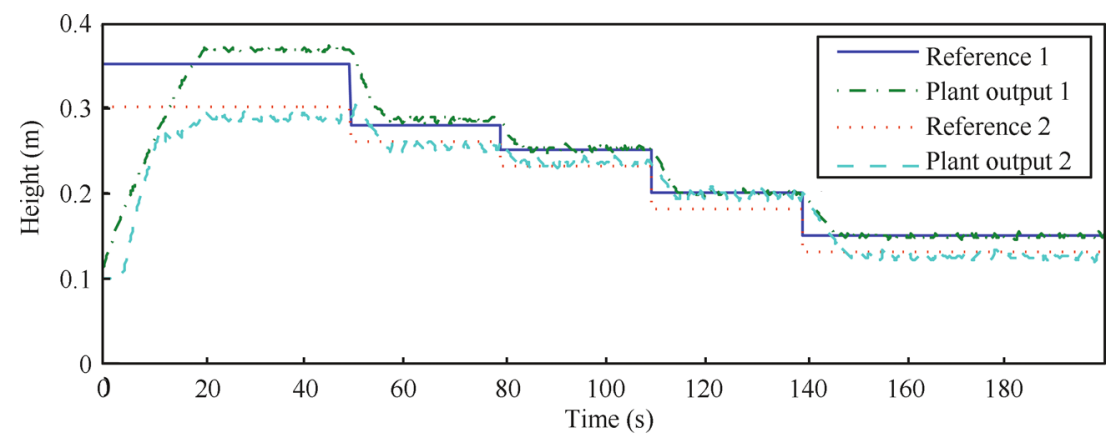

(a) ANN

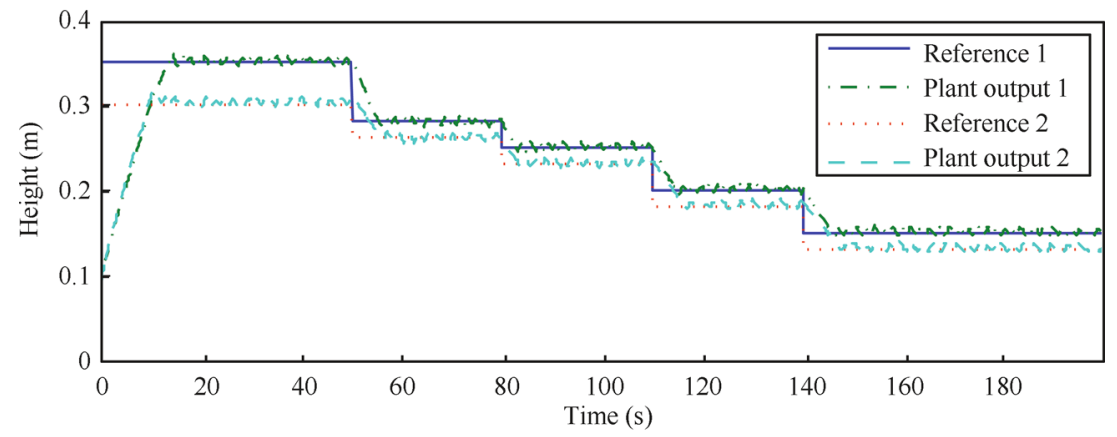

(b) WNN

Fig. 10 Real time NMPC strategy tracking different heights

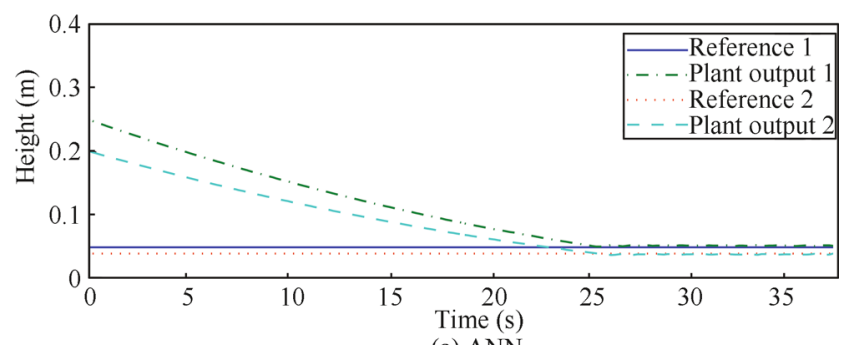

(a) ANN

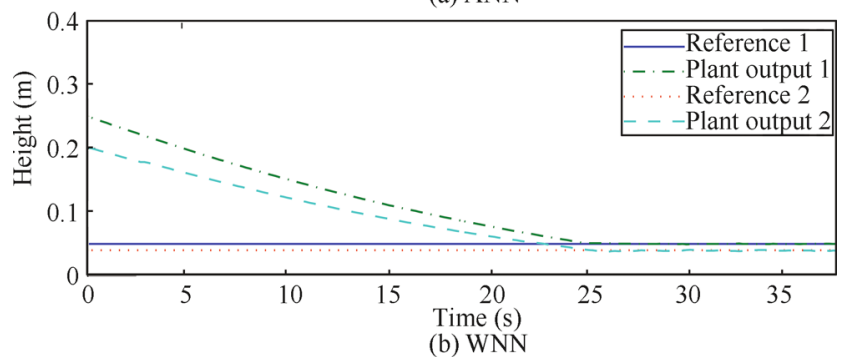

Fig. 11 Abnormal NMPC Strategy scenarios tracking low level in simulation

This same above scenario is implemented in real time. Figs. 14 (a) and (b) show the real time performances of NMPC with both ANN and WNN models in the abnormal situation of tracking high height. Just as in the simulation case, the ANN model could not maintain both the levels while $\mathrm{WNN}$ is able to track the $0.2 \mathrm{~m}$. Both levels are the same here because of the applied maximum interaction. The MSE obtained for WNN is $1.4 \times 10^{-4} \mathrm{~m}^{2}$ while ANN is $0.0048 \mathrm{~m}^{2}$. The ACE obtained for ANN is less because it is doing lesser useful work as explained already in the simulation case. This also shows that WNN performed better than ANN in both simulation and real time case.
Moreover, the results indicate that the WNN model not only perform better than the ANN model but also can operate effectively in abnormal situations which can arise at any time because of plant degradations, valve malfunctions and equipment wear and tear. This is one of the benefits of using non-linear models even though the NMPC strategy is not working adaptively. The results given in the abnormal scenarios show that the non-linear model predictive controller can adapt easily as it takes action based on the present situation. The NMPC can adopt easily as the real system is not a $100 \%$ match to the simulated system but results confirm that robust control is achieved in this 

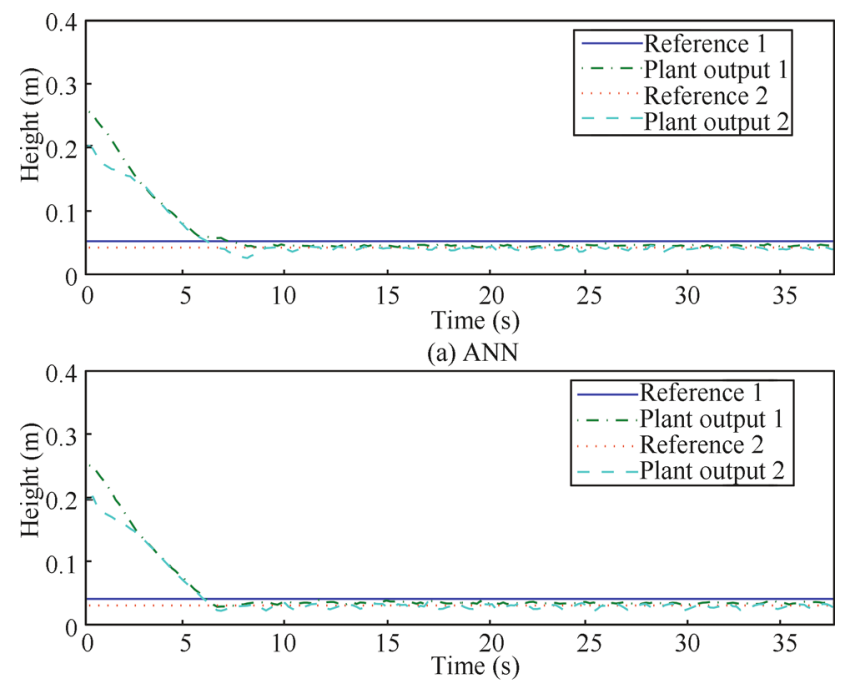

(b) WNN

Fig. 12 Abnormal NMPC strategy scenarios tracking low level in real time

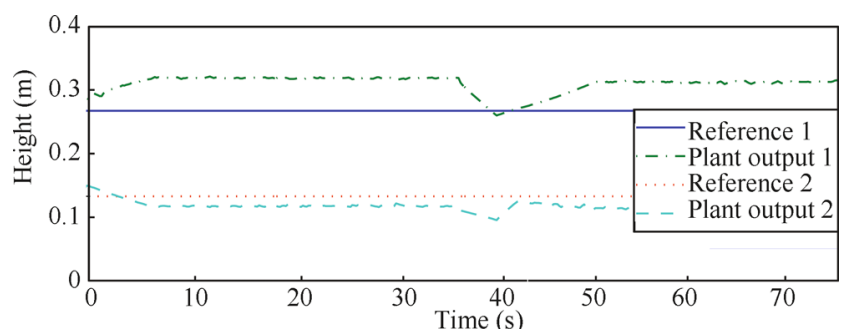

(a) ANN

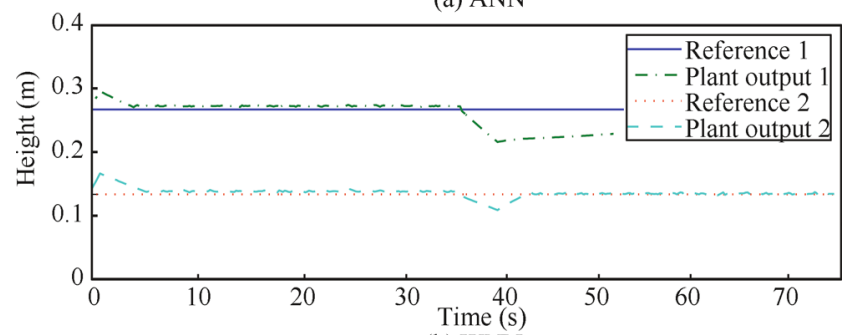

(b) WNN

Fig. 13 Abnormal NMPC strategy scenarios tracking high level in simulation

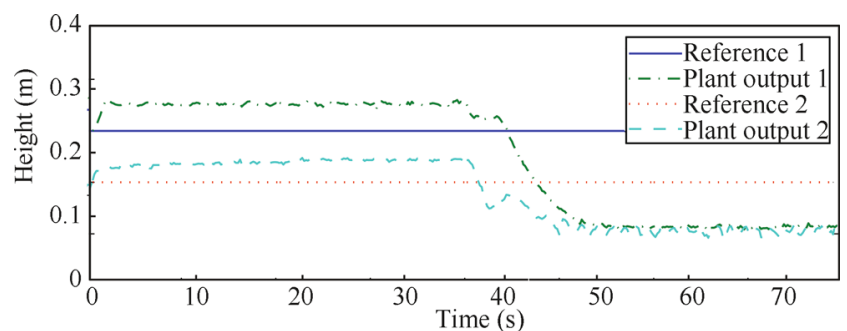

(a) ANN

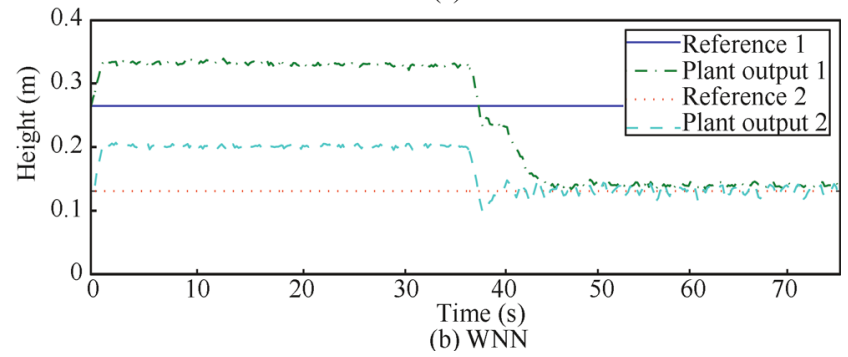

Fig. 14 Abnormal NMPC strategy scenarios tracking high level in real time 
case as well. The paper considers different scenarios to take into account of abnormal situations. Real time application of GA helps to achieve optimised controller parameters in the non-linear case, which is difficult to obtain by other means.

\section{Conclusions}

This paper has demonstrated a novel model based WNNNMPC strategy for an MIMO CTS and implemented both in simulation and in real time. A system identification approach was employed by training raw input-output data obtained from open loop experiment. In order to handle the difficulties in network training, a global search stochastic wavelet conjugate algorithm is employed for initial network training to give a good initial starting weight for training a GA. This is a case of non-linear MIMO system, which is more challenging than most recently reported SISO in the literature, and this training approach was deployed in order to avoid the problem of being trapped in a local minimum

\section{References}

[1] R. Findeisen, F. Allgower. An introduction to non-linear model predictive control. Information and Control, vol. 11, no. 1-2, pp. 1-23, 2002.

[2] D. E. Seborg, T. F. Edgar, D. A. Mellichamp, F. J. Doyle III. Process Dynamics and Control, 3rd ed., Inc United States: John Wiley Sons, 2011.

[3] E. Laubwald. Coupled tank system. Control Systems Principles, pp. 1-8, 2005.

[4] K. Owa, S. K. Sharma, R. Sutton. Non-linear model predictive control strategy based on soft computing approaches and real time implementation on a coupled-tank system. International Journal of Advanced Research in Computer Science and Software Engineering, vol. 3, no. 5, pp. 1350-1359, 2013.

[5] W. Grega, A. Maciejczyk. Digital control of a tank system. IEEE Transactions on Education, vol. 37, no. 3, pp. 271276, 1994.

[6] S. M. Nawi, A. N. Abdalla, M. S. Ramli. Improved coupled tank liquid levels system based on hybrid genetic-immune adaptive tuning of PI controller. In Proceedings of the International Conference on Electrical, Control and Computer Engineering, IEEE, Pahang, Malaysia, pp. 247-252, 2011.

[7] M. U. Khalid, M. B. Kadri. Liquid level control of non-linear coupled tanks system using linear model predictive control. In Proceedings of International Conference on Emerging Technologies, no. 1, pp. 1-5, 2012.

[8] I. Holič, V. Vesely. Robust PID controller design for coupled-tank process. In Proceedings of the 18th International Conference on Process Control, Tatranska Lomnica, Slovakia, pp. 506-512, 2011.

[9] V. Ramakrishnan, Y. Zhuang, S. Y. Hu, J. P. Chen, C. C. Ko, B. M. Chen, K. C. Tan. Development of a web-based solution. Initial heuristic results showed that the proposed two-stage training is a faster approach. The obtained reliable non-linear model of the CTS showed the effectiveness of the system identification procedure which allows for a wide range of prediction capabilities. Results further showed that the single WNN model is well suitable to perform in all operating regions of control and the capabilities of handling disturbance rejection. It was also shown that WNN model could perform even at abnormal conditions especially in cases of plant degradation, valve malfunctions, and equipment wear. This shows the strength of the WNN non-linear model in tackling difficult MIMO problems especially when properly trained without the use of adaptive models. Furthermore, the whole NMPC strategy is able to overcome the challenges of using GA for RTO and real time applications for real-time set point tracking. This is also evident in that the sampling time of $0.2 \mathrm{~s}$ is smaller compared to most reports in cited literature. The whole strategy is well suited for chemical processes with varying interaction rates.

control experiment for a coupled tank apparatus. In Proceedings of American Control Conference, Chicago, Illinois, USA, pp. 4409-4413, 2000.

[10] M. F. Rahmat, S. M. Rozali. Modeling and controller design for a coupled-tank liquid level system: Analysis and comparison. Jurnal Teknology, vol. 48, no. D, pp. 113-141, 2008 .

[11] M. Kubalcik, V. Bobal. Adaptive control of three-tanksystem: Comparison of two methods. In Proceedings of the 16th Mediterranean Conference on Control and Automation, IEEE, Ajaccio, pp. 1041-1046, 2008.

[12] M. Majstorovic, I. Nikolic, J. Radovic, G. Kvaščev. Neural network control approach for a two-tank system. In Proceedings of the 9th Symposium on Neural Network Applications in Electrical Engineering, IEEE, Belgrade, Serbia, pp. 215-218, 2008.

[13] I. Alvarado, D. Limon, A. Ferramosca, E. F. Camacho. Robust tubed-based MPC for tracking applied to the quadruple-tank process. In Proceedings of the 17th IEEE International Conference on Control Applications, IEEE, San Antonio, Texas, USA, vol. 3, no. 3, pp. 305-310, 2008.

[14] TecQuipment. CE105MV. Control Engineering, Sensors and Instrumentation, pp. 1-3, 2013.

[15] K. Owa, S. K. Sharma, R. Sutton. Optimised multivariable non-linear predictive control for coupled tank applications. In Proceedings of the 1st IET Control and Automation Conference, Conference Aston Lakeside Centre, IET, Birmingham, UK, pp. 1-6, 2013.

[16] T. Tani, S. Murakoshi, M. Umano. Neuro-fuzzy hybrid control system of tank level in petroleum plant. IEEE Transactions on Fuzzy Systems, vol. 4, no. 3, pp. 360-368, 1996.

[17] B. Guo, A. Jiang, X. Hua, A. Jutan. Non-linear adaptive control for multivariable chemical processes. Chemical Engineering Science, vol. 56, no. 23, pp. 6781-6791, 2001. 
[18] M. Senthilkumar, S. A. Lincon. Design of stabilizing PI controller for coupled tank MIMO process. International Journal of Engineering Research and Development, vol. 3, no. 10 , pp. $47-55,2012$.

[19] K. A. Kosanovich, M. J. Piovoso, V. Rokhlenko, A. Guez. Non-linear adaptive control with parameter estimation of a CSTR. Journal of Process Control, vol. 5, no. 3, pp. 137$148,1995$.

[20] C. Tricaud, Y. Q. Chen. Linear and non-linear model predictive control using a general purpose optimal control problem solver RIOTS 95. In Proceedings of the $2008 \mathrm{Chi}-$ nese Control Decision Conference, IEEE, Yantai, China, pp. 1552-1557, 2008.

[21] T. Y. Chai, Y. J. Zhang, H. Wang, C. Y. Su. Data-based virtual unmodeled dynamics driven multivariable non-linear adaptive switching control. IEEE Transactions on Neural Networks, vol. 22, no. 12, pp. 2154-2172, 2011.

[22] R. Jain, T. Vinoprabha, N. Sivakumaran, T. K. Radhakrishnan. Design and implementation of controllers for MIMO process. In Proceedings of 2009 International Conference on Advances in Recent Technologies in Communication and Computing, IEEE, Kottayam, Kerala, India, vol. 1, pp. 750-752, 2009.

[23] J. Richalet. Industrial applications of model based predictive control. Automatica, vol. 29, no. 5, pp. 1251-1274, 1993.

[24] H. Zhao, J. Guiver, R. Neelakantan, L. T. Biegler. A non-linear industrial model predictive controller using integrated PLS and neural net state-space model. Control Engineering Practice, vol. 9, no. 2, pp. 125-133, 2001.

[25] M. A. S. Al-Qaisy. Linear and non-linear multi-input multioutput model predictive control of continuous stirred tank reactor. Tikrit Journal of Engineering Science, vol. 19, no. 3, pp. 41-57, 2012.

[26] T. Heckenthaler, S. Engell. Approximately time-optimal fuzzy control of a two-tank system. IEEE Control Systems Magazine, vol. 14, no. 3, pp. 24-30, 1994.

[27] A. A. R. Diniz, P. R. M. Pires, J. D. de Melo, A. D. D. Neto, A. J. J. L. Filho, S. M. Kanazava. Reinforcement learning for controlling a coupled tank system based on the scheduling of different controllers. In Proceedings of the 2010 Eleventh Brazilian Symposium on Neural Networks, IEEE, Sao Paulo, Brazil, pp. 212-216, 2010.

[28] M. Senthilkumar, D. S. AbrahamLincon, P. Selvakumar. Design of PI controller using characteristic ratio assignment method for coupled tank SISO process. International Journal of Computer Applications, vol. 25, no. 9, pp. 49-53, 2011.

[29] A. G. Ram, S. A. Lincoln. A model reference-based fuzzy adaptive PI controller for non-linear level process system. International Journal of Research and Reviews in Applied Sciences, vol. 14, no. 2, pp. 477-486, 2013.

[30] A. Maalla, C. Wei, M. H. Hafiz. Model parameter identification of a coupled industrial tank system based on a wavelet neural network. Dcabes 2008 Proceedings, Vols I II, pp. 1251-1254, 2008 .
[31] Y. Oussar, I. Rivals, L. Personnaz, G. Dreyfus. Training wavelet networks for non-linear dynamic input-output modelling. Neurocomputing, vol. 20, no. 1-3, pp.173-188, 1998.

[32] D. Huang, Y. Wang, Y. Jin. Non-linear MIMO Adaptive Predictive Control Based on Wavelet Network Model. In Proceedings of International Symposium on Advanced Control of Chemical Processes, IFAC, Hongkong, China, 2004.

[33] C. J. Lin, C. C. Chin, C. L. Lee. A wavelet-based neurofuzzy system and its applications. In Proceedings of the International Joint Conference on Neural Networks, IEEE, Portland, OR, USA, pp. 1921-1926, 2003.

[34] F. Jajangiri, A. Doustmohammadi, M. B. Menhaj. Identification of twin-tanks dynamics using adaptive wavelet differential neural networks. In Proceedings of International Joint Conference on Neural Networks, IEEE, Barcelona, Spain, pp. 1-5, 2010.

[35] J. C. Lu, Z. H. Gu, H. Q. Wang. Research on the application of the wavelet neural network model in peak load forecasting considering the climate factors. In Proceedings of 2005 International Conference on Machine Learning and Cybernetics, IEEE, Guangzhou, China, pp. 538-543, 2005.

[36] M. Meng, W. Sun. Short-term load forecasting based on rough set and wavelet neural network. International Conference on Computational Intelligence and Security, IEEE, Suzhou, China, pp. 446-450, 2008.

[37] Y. R. Kuraz. Design of multi wavelet network identifier for multi input multi output nonlinear functions. In Proceedings of International Arab Conference on Information Technology, vol. 2, pp. 7-9, 2006.

[38] L. L. Wei, D. F. Xu, H. Sun, M. X. Zhou. Application of optimized wavelet neural network based on genetic algorithm in stock market prediction. China Computer and Communication, vol. 11, n5.1, pp.130-131, 2001. (in Chinese)

[39] D. Coca, S. A. Billings. System identification using wavelets. Control Systems Robotics and Automation, vol. 6, pp. 1-10, 2012.

[40] C. M. Leavey, M. N. James, J. Summerscales, R. Sutton. An introduction to wavelet transforms: A tutorial approach. Insight, vol. 45, no. 5, pp. 344-353, 2003.

[41] F. Jahangiri, A. Doustmohammadi, M. B. Menhaj. An adaptive wavelet differential neural networks based identifier and its stability analysis. Neurocomputing, vol.77, no. 1 , pp. 12-19, 2012.

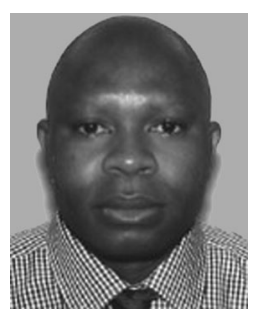

Kayode Owa received his B. Sc. degree in computer science and engineering and obtained his M. Sc. degree in computer science (process control). He was awarded his Ph. D. degree from the Plymouth University, UK in 2014. His research studies involve wavelet based computational nonlinear modelling, advanced model based predictive control strategy for real time applications, and non-linear real time optimisation approaches. He has published papers in the field of non-linear model based predictive control strategies for coupled tank applications. He has 
also participated as a reviewer in peer-reviewed papers for reputable international journals such as the IEEE Intelligent Transportation Systems Society, and International Journal of Automation and Computing. He is currently a member of Marine and Industrial Dynamic Analysis (MIDAS) Research Group.

His research interests include various aspects of system identification and modelling, advanced model based control systems and applications, real time practical implementation, and multiinput multi-output systems, real time optimisation, and artificial intelligent techniques such as particle swarm optimisation, genetic algorithms, and neural networks.

E-mail: kayode.owa@plymouth.ac.uk, olayemi_owa@yahoo.co.uk (Corresponding author)

ORCID iD: 0000-0002-1393-705X

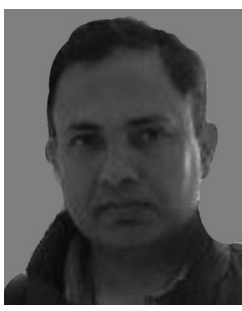

Sanjay Sharma is a lecturer in the School of Marine Science and Engineering and head of the Marine and Industrial Dynamic Analysis (MIDAS) Research Group. He worked as a signal and telecommunication engineer in Indian Railways for four and a half years and was involved with route relay and solid state interlocking design projects. Prior to joining Plymouth University in 2004, he held a position as a research engineer in the Intelligent Systems and Control Research Group at Queen University of Belfast, UK.

His research interests include the application of soft computing techniques in optimisation, fault diagnosis and parallel computing for neural and local model network training, evolutionary approaches to multiple-modelling and control of non-linear industrial and marine systems.

E-mail: sanjay.sharma@plymouth.ac.uk

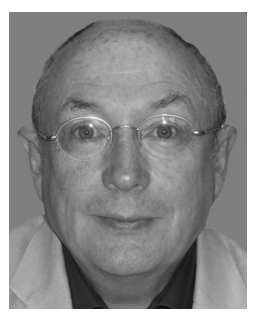

Robert Sutton holds the degrees of B. Eng. (Tech.) in engineering production, and M. Eng. and Ph. D. degrees in control engineering from the University of Wales, UK. Prior to joining the Royal Navy as a commissioned officer in 1976, he served general engineering and student apprenticeships with Firth Cleveland Fastenings Limited, Pontypridd, UK, followed by a period as a research student in the Industrial Engineering Unit, UWIST, Cardiff. On completion of his service in 1992 in the rank of Lieutenant Commander Royal Navy, he took up an appointment at the University of Plymouth. Until recently, he was a head of School and is currently professor of control systems engineering in the School of Marine Science and Engineering. He is also a member of the Marine and Industrial Dynamic Analysis Research Group within the University.

In addition, he is a member of the International Federation of Automatic Control Technical Committee on Marine Systems and the Society for Underwater Technology Underwater Robotics Group Committee. He is the author/co-author of over 180 book, journal and conference publications. On six occasions, he has been the recipient of premier awards from institutions for the most outstanding technical paper appearing in their journal for a given year.

His research interests include the application of advanced control engineering and artificial intelligence techniques to control problems. Ongoing research is concerned with the use of fuzzy logic, neurofuzzy algorithms, artificial neural networks and adaptive search algorithms in the design of novel control systems for industrial and marine plant.

E-mail: R.Sutton@plymouth.ac.uk 